\title{
$\underline{\mathbf{P}-45}$
}

\section{Phytochemical Studies of Malaysian Crotalaria Mucronata}

\author{
Ann-chee Yap ${ }^{*}$ and Yeun-mun Choo \\ Department of Chemistry, Faculty of Science, University of Malaya, 50603 Kuala Lumpur, Malaysia; \\ E-mail: cady.yap@gmail.com
}

Crotalaria $s p$. is a perennial subshrub-like herb with yellow flowers and leguminous pod. It has been shown to produce bioactive secondary metabolites and is often associated with livestock poisoning. The seeds of Crotolaria sp. contain pyrrolizidine alkaloids such as monocrotaline. Despite the toxicity nature of the plant, it has been in traditional medicine for the anti-inflammatory treatment. Previous study on Crotolaria $s p$. from other countries has reported the isolation of flavonoids, chalcones, terpenes, lectins, and polysaccharides. However, the phytochemical of the Malaysia Crotolaria has not been studied before. In the present study, Crotolaria mucronata has been collected from the hilly area in the Pahang state in Malaysia. The whole plant was dried and grounded and followed by ethanol extraction. The ethanolic crude extract was further partitioned into hexane, chloroform, and ethanol. The hexane extract was subjected to extensive chromatographic separation and the compounds isolated are characterized by various spectroscopic methods such as Nuclear Magnetic Resonance and mass spectrometry. The compounds identified thus far are unsaturated fatty acids, fatty acid esters, a straight chain alkane and 5hydroxy-3,4-dimethyl5-pentylfuran-2(5H)-one.

Keywords: Fabaceae, Crotalaria Mucronata, Fatty acids, Alkane, Furanone. 